\title{
Lidil
}

Revue de linguistique et de didactique des langues

$62 \mid 2020$

Recherches actuelles en didactique du lexique : avancées, réflexions, méthodes

\section{La discussion lexicale : une approche dialogique pour l'analyse des relations sémantiques}

Lexical Discussion: A Dialogical Approach for Semantic Relationships Analysis

\section{Constance Lavoie, Patricia-Anne Blanchet et Martine Pellerin}

\section{OpenEdition}

\section{Journals}

Édition électronique

URL : http://journals.openedition.org/lidil/8456

DOI : $10.4000 /$ lidil.8456

ISSN : 1960-6052

Éditeur

UGA Éditions/Université Grenoble Alpes

Édition imprimée

ISBN : 978-2-37747-226-0

ISSN : $1146-6480$

Référence électronique

Constance Lavoie, Patricia-Anne Blanchet et Martine Pellerin, « La discussion lexicale : une approche dialogique pour l'analyse des relations sémantiques », Lidil [En ligne], 62 | 2020, mis en ligne le 03 novembre 2020, consulté le 05 novembre 2020. URL : http://journals.openedition.org/lidil/8456 ; DOI : https://doi.org/10.4000/lidil.8456

Ce document a été généré automatiquement le 5 novembre 2020

(C) Lidil 


\section{La discussion lexicale : une approche dialogique pour l'analyse des relations sémantiques}

Lexical Discussion: A Dialogical Approach for Semantic Relationships Analysis

Constance Lavoie, Patricia-Anne Blanchet et Martine Pellerin

\section{Introduction}

1 L'apprentissage des relations sémantiques est une tâche complexe qui nécessite de tisser des liens de sens en partant de mots connus et des représentations initiales des apprenants (Vygotsky, 1987). Selon Polguère (2016), cet apprentissage devrait s'articuler autour des trois facettes qui composent le lexique: la forme, le sens et l'usage. Or, de façon générale, peu d'enseignement systématique du lexique serait réalisé en classe (Dreyfus, 2004) et le potentiel des approches discursives pour cet apprentissage serait peu exploité (Calaque, 2000). En effet, la plupart des enseignants priorisent l'apprentissage de l'orthographe (la forme) et le font surtout par l'écrit à partir d'exercices ponctuels et de listes orthographiques prescrites (Anctil, 2019; Calaque, 2000). L'étude d'Anctil, Singcaster et Tardif (2018) révèle que les pratiques les plus courantes dans les classes primaires au Québec sont l'explication du sens de mots de la liste orthographique hebdomadaire et lors d'activités de lecture en grand groupe. Les enseignantes du $2^{\mathrm{e}}$ et du $3^{\mathrm{e}}$ cycle du primaire au Québec déclarent que $28,8 \%$ du temps consacré à l'enseignement lexical porte sur les relations entre les mots. Ces auteurs parlent de "discussions lexicales incidentes» pour qualifier ces courts échanges ponctuels non planifiés visant à expliquer le sens d'un mot. Ils précisent que les discussions lexicales incidentes constituent $25,3 \%$ des activités lexicales déclarées par les enseignantes du $2^{\mathrm{e}}$ cycle du primaire. Ces résultats corroborent ceux de Dreyfus (2004), qui indique que les discussions à teneur lexicale ne mènent pas à des traces ni à des réinvestissements. Ainsi, la dimension discursive serait toujours peu exploitée pour travailler systématiquement le sens des mots et le lexique en général 
(Bengochea, 2014 ; Calaque, 2000). Par conséquent, notre étude visait à développer une démarche didactique mariant la discussion lexicale à l'enseignement explicite de notions lexicales. Or, les notions lexicales n'ont pas toutes le même niveau de complexité. Par exemple, expliquer le lien de synonymie implique la maitrise des classes de mots et du sens, puisque deux synonymes doivent être de la même classe de mots et être permutables (Amer, 2002 ; Duvignau \& Garcia-Debanc, 2008; Edmonds \& Hirst, 2002).

Devant le manque de ressources didactiques informées par des approches dialogiques pour l'enseignement du lexique et en réponse aux prescriptions ministérielles, il apparaissait pertinent de développer une démarche axée sur le dialogue. Cette démarche didactique pour l'enseignement des relations sémantiques s'intitule la communauté de recherche lexicale (CRL). Pour favoriser le partage des outils didactiques mis au point dans le cadre de ce projet de recherche, une ressource en ligne a été créée et est accessible à tous sur le site de la Communauté de recherche lexicale à l'adresse <http://crl.recherche.usherbrooke.ca/>.

La CRL se définit comme une démarche didactique dialogique pour l'enseignement et l'apprentissage des relations sémantiques aussi appelées liens de sens (Lavoie, Pellerin \& Girard, 2017). Elle est conçue pour les élèves du primaire âgés de 8 à 12 ans, puisque les notions de liens de sens (mot de même famille morphologique, mot en lien avec une thématique, synonymie, antonymie) sont prescrites au programme pour cette tranche d'âge au Québec (MELS, 2011). Concrètement, la CRL comporte cinq étapes qui consistent à accompagner progressivement les élèves dans le développement de stratégies lexicales et métacognitives pour apprendre à repérer et à analyser les relations qui existent entre les mots (Lavoie, Pellerin \& Girard, 2017). La figure 1 présente ces cinq étapes. 
Figure 1. - Les étapes d'une communauté de recherche lexicale.

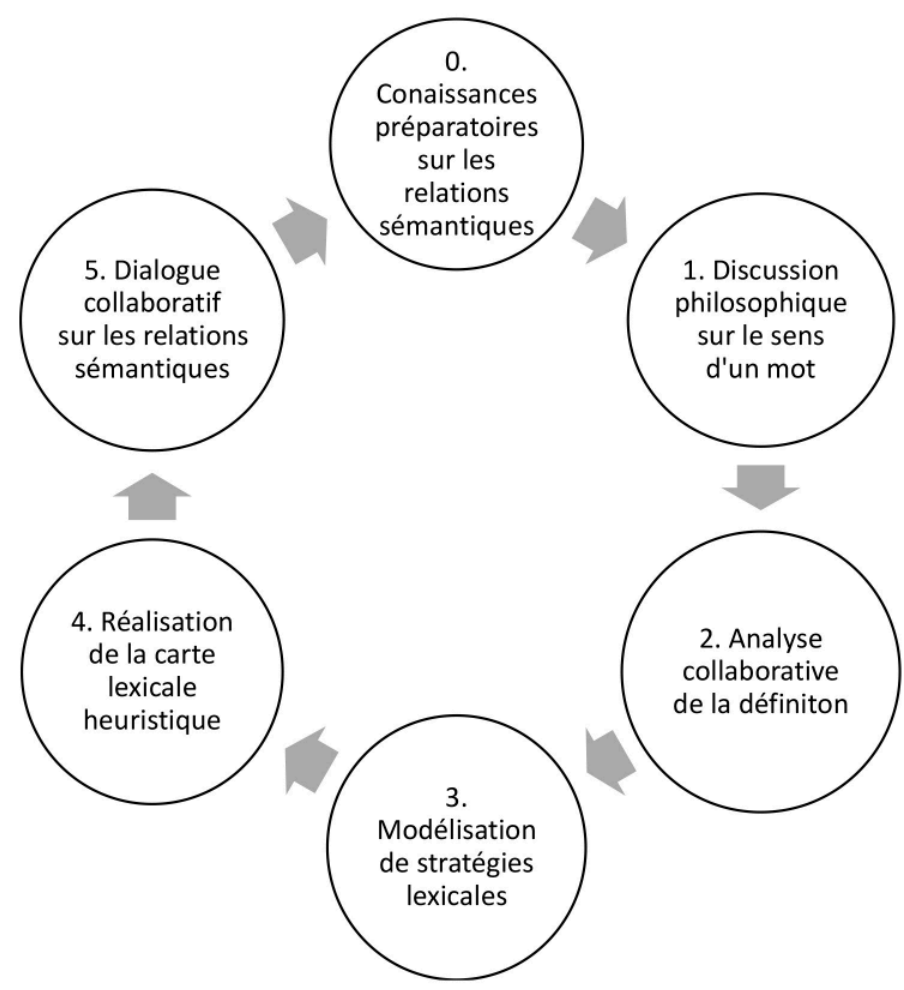

4 La première étape ${ }^{2}$ consiste à engager les apprenants dans une discussion lexicale sur un mot connu de tous les élèves durant laquelle la moitié d'entre eux réfléchissent au sens d'un mot et en discutent tandis que l'autre moitié écoute et note les mots entendus ayant un lien de sens avec le mot dont il est question durant la discussion. La seconde étape $^{3}$ prévoit l'analyse collaborative de la définition du mot. Lors de cette étape, les élèves élaborent seuls, puis en dyades et finalement en groupe leur définition avant d'en révéler le sens proposé dans le dictionnaire et de distinguer, si c'est le cas, les différents sens du mot. La troisième étape ${ }^{4}$ consiste à faire un enseignement explicite de stratégies lexicales pour établir les relations sémantiques entre les mots notés durant la discussion lexicale réalisée lors de la première étape. L'enseignante modélise alors les stratégies lexicales à l'aide de l'arbre de décisions lexicales ${ }^{5}$. L'étape quatre correspond à la réalisation par chaque élève d'une carte lexicale heuristique ${ }^{7}$. S'ensuit, lors de la dernière étape ${ }^{8}$, un dialogue collaboratif sur les diverses relations sémantiques établies par le groupe.

Cet article explore de façon plus approfondie la première étape de la démarche relative à la discussion lexicale sur le sens d'un mot et l'écoute active des mots. Il répond à la question suivante : en quoi une discussion lexicale réalisée par des élèves de 8-9 ans en contexte scolaire plurilingue au Québec dans le cadre de la CRL est-elle suffisamment riche lexicalement pour amorcer un travail d'analyse des relations sémantiques? Afin de répondre à cette question, les principales théories et approches impliquées dans l'élaboration de la CRL doivent être définies. 


\section{Cadrage théorique}

6 Le développement de la démarche didactique de la CRL prend appui sur la théorie socioculturelle des interactions ainsi que sur les approches discursives. Il s'inspire d'avancées réalisées dans le champ de la philosophie pour enfants.

\subsection{Théorie socioculturelle des interactions}

7 La théorie socioculturelle de Vygotsky (1987) confère un rôle central au dialogue dans la constitution de la réalité sociale et du développement lexical. Celle-ci offre une base théorique pour conceptualiser le lien entre l'interaction, en tant qu'activité sociale, et l'acquisition du lexique, en tant qu'activité sociocognitive. Cette théorie soutient qu'il est préférable d'apprendre des mots en contexte d'interaction que d'apprendre des mots de façon isolée et conçoit que le développement langagier se coconstruit durant les échanges entre les apprenants (Mondada \& Pekarek Doehler, 2004). Dans cette perspective socioconstructiviste, la prise de parole en contexte d'interaction constitue une opération cognitive essentielle au développement de la "pensée verbale ». Selon Vygotsky (1987), il ne faut pas viser l'acquisition immédiate du sens d'un mot, mais travailler le mot comme le constituant de cette "pensée verbale» (p. 321), à travers une démarche favorisant l'interaction entre la pensée et l'apprentissage lexical. Pour ce dernier, la verbalisation, combinée à l'écoute des pairs, ferait évoluer la compréhension sémantique des mots. Goodwin et Kyratzis (2007) soutiennent en ce sens que l'interaction se développe grâce à la mutuelle réflexivité des locuteurs et des observateurs en tant qu'acteurs orientant les échanges. Qui plus est, la théorie socioculturelle tient compte de différentes formes de participation active durant les interactions et ne se limite pas à la prise de parole. Ainsi, l'écoute, l'observation, les réactions non verbales et la production d'artéfacts sont toutes des manifestations de participation active à la trame discursive (Van Compernolle, 2015). Dans une CRL, cette participation active se révèle par la verbalisation des élèves interlocuteurs, la prise de note des élèves observateurs et leur écoute active de la discussion.

8 Par ailleurs, les travaux de Firth et Wagner (1997) ainsi que ceux de Mondada et Pekarek Doehler (2004) rappellent que le niveau des compétences langagières est indissociable de la pluralité des contextes sociaux et culturels dans lesquels les apprenants vivent. Il est connu que les enfants provenant des milieux économiquement défavorisés ont un vocabulaire plus limité que les enfants provenant de milieux socioénonomiquement favorisés (Hart \& Risley, 1995). Ainsi, le contexte influence l'interaction (Van Compernolle, 2015) et, par extension, le bagage lexical et les interactions des locuteurs influencent le contexte des échanges (Vygotsky, 1987). En raison de la pluralité des expériences et des représentations, il est donc préférable d'enseigner la signification d'un mot en partant du bagage lexical des apprenants (Mondada \& Pekarek Doehler, 2004). En effet, ceux-ci possèdent déjà une représentation des mots, laquelle est déterminée par l'âge, le milieu socioéconomique, la personnalité, les origines culturelles et la langue première. Par conséquent, l'écoute des représentations est nécessaire pour accompagner les élèves dans leur apprentissage des relations sémantiques. 


\subsection{Approches discursives pour l'apprentissage du lexique}

9 Les approches discursives s'inscrivent dans un continuum avec la théorie socioculturelle, puisque tous deux placent l'interaction verbale comme pilier central pour l'enseignement-apprentissage du lexique. Mazière $(1993)$, Nonnon $(1996,2008)$ et Plane et Lafourcade (2004) défendent la pertinence de ces approches, puisque la pratique de la discussion favorise l'émergence de la multiplicité référentielle des mots. Les mots ne s'apprennent pas isolément, chacun dans leur rapport à un seul référent, mais dans les échos qu'ils ont les uns avec les autres (Aitchison, 2012), dans la cohérence sémantique et énonciative d'une unité plus large permettant un éclairage sur les caractéristiques du référent. Dans le domaine de la didactique générale, les approches discursives consistent à proposer une "tâche discursive » (Grandaty, 2006) qui implique que l'enseignante guide les élèves dans la gestion d'un acte collectif de parole et d'écoute. Grandaty (2006) caractérise les tâches discursives comme des tâches qui orientent l'activité langagière, favorisent les échanges entre pairs, impliquent des négociations et des prises de conscience sur l'activité disciplinaire et transforment les postures des apprenants. Cet article examine l'étendue lexicale déployée par les élèves lors de la tâche discursive dans le cadre de la première étape de la CRL, qui constitue l'amorce d'un travail d'analyse des relations sémantiques.

\subsection{Discussion philosophique et discussion lexicale}

10 Le déroulement des discussions lexicales s'inspire des discussions philosophiques conduites lors des communautés de recherche philosophique (CRP) créées par les philosophes et pédagogues Lipman, Sharp et Oscanyan(1980), sous le nom de Community of Philosophical Inquiry (CPI). D'ailleurs, le nom de la démarche didactique des communautés de recherche lexicale (CRL) est tiré de ce modèle pédagogique. La discussion philosophique préconise l'autonomie de l'enfant et la pratique du dialogue (Sasseville \& Gagnon, 2012). La démarche pédagogique de la CRP n'est pas réservée à la discipline de la philosophie, et les critères sur lesquels elle repose peuvent être transposés à diverses matières scolaires. À titre d'exemple, elle a déjà été adaptée à l'enseignement des mathématiques, des sciences (Daniel, Lafortune, Pallascio \& Sykes, 2004) et de l'éthique et de la culture religieuse à l'école (Gagnon, 2012). Cette étude s'est inspirée de la CRP pour certaines conduites discursives, l'aménagement de la classe ainsi que le rôle attendu de l'enseignante pour guider la discussion.

\subsection{Déroulement d'une discussion lexicale}

11 La discussion lexicale correspond à la première étape de la CRL. Par l'entremise d'une activité d'amorce ou d'un déclencheur qui capte l'attention (musique, image, objet, lecture, vidéo, etc.), l'enseignante amorce une discussion sur le sens d'un mot donné. Par exemple, une vidéo d'une expérience scientifique peut servir d'amorce pour la discussion sur le sens du mot RECHERCHER. D'un point de vue de l'aménagement de la classe, le groupe-classe est séparé en deux: une partie composée d'élèves interlocuteurs qui discutent en cercle et une autre composée d'élèves observateurs qui écoutent activement les échanges à l'extérieur du cercle en prenant en note sur une carte les mots entendus ayant, à leur avis, un lien de sens avec le mot du jour. 
Figure 2. - Aménagement physique d'une discussion lexicale.

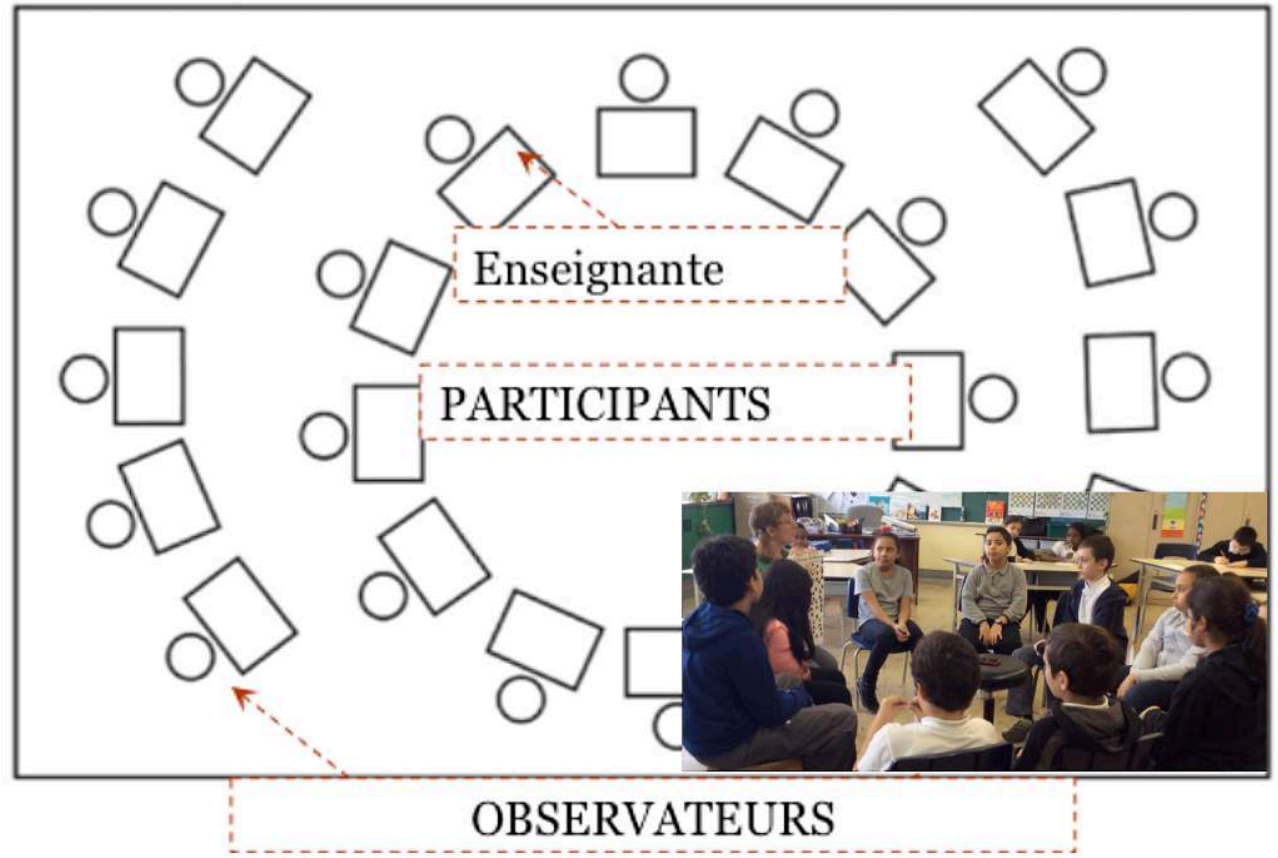

12 Afin de maximiser l'attention des participants, chaque discussion lexicale n'excède pas quinze minutes, au terme desquelles les élèves alternent leurs rôles. Les élèves interlocuteurs deviennent ainsi observateurs et vice versa. La discussion lexicale est captée sur vidéo afin de permettre des visionnements ultérieurs pour enrichir l'analyse des mots.

$13 \mathrm{Au}$ cours de la discussion lexicale, le rôle de l'enseignante est de stimuler, par son écoute active, ses reformulations et ses questionnements, la compréhension et le raffinement des relations sémantiques des élèves. Il ne s'agit pas de donner une définition du mot, mais de les encourager à établir des liens de sens. Durant la discussion, l'enseignante guide les échanges par des questionnements prédéterminés dans un guide de discussion ${ }^{9}$, qui offre des questions que l'enseignante mobilise ou non en fonction des propos des apprenants et de l'orientation de la discussion. Le tableau 1 présente des questions types auxquelles l'enseignante peut recourir afin de stimuler la discussion autour de chaque catégorie de relation sémantique. 
Tableau 1. - Questions types pour susciter la discussion lexicale sur les liens de sens.

\begin{tabular}{|c|c|c|}
\hline Mot lié à une thématique & \multicolumn{2}{|r|}{ Mot de même famille morphologique } \\
\hline $\begin{array}{l}\text { À quel thème ou catégorie de mots } \mathrm{X} \\
\text { et Y sont-ils reliés? } \\
\text { Q Quel est le sens partagé entre le mot } \mathrm{X} \\
\text { et le mot } Y \text { ? } \\
\circ \quad \text { Peux-tu donner un exemple de mot } \mathrm{X} \text { ? }\end{array}$ & ० & $\begin{array}{l}\text { Quels autres mots de la même famille } \\
\text { que le mot X connaissez-vous? } \\
\text { Peut-on faire un mot plus long avec le } \\
\text { mot } X \text { ? }\end{array}$ \\
\hline Synonyme & & Antonyme \\
\hline $\begin{array}{l}\text { Quel mot peut exprimer la même idée } \\
\text { que le mot Y? } \\
\text { Est-ce que le mot Y veut dire la même } \\
\text { chose que le mot X? } \\
\text { Peux-tu essayer de les substituer dans } \\
\text { la phrase entendue? }\end{array}$ & $\begin{array}{l}0 \\
0\end{array}$ & $\begin{array}{l}\text { Quel mot est l'inverse du mot } X \text { ? } \\
\text { Peux-tu trouver un mot plutôt que la } \\
\text { négation du mot } X \text { ? }\end{array}$ \\
\hline
\end{tabular}

Tiré de Lavoie, Pellerin \& Girard (2017, p. 16).

14 Les guides de discussion ont été élaborés en reprenant plusieurs critères observés en CRP dans les travaux de Sasseville et Gagnon (2012). Ces critères ont été adaptés afin qu'ils se conforment plus précisément à la recherche lexicale et qu'ils stimulent la réflexion des élèves relativement au sens des mots. Ces critères sont en quelque sorte des outils de la pensée généralement utilisés dans l'exercice d'argumentation (Sasseville \& Gagnon, 2012). Parmi ceux-ci ont été retenus le doute, la comparaison, les contre-exemples, la nuance et la contextualisation.

Le doute constitue un critère en CRP qui permet de remettre en question une partie, voire l'ensemble des croyances de l'élève. Dans la CRL, le doute est suscité chez l'apprenant afin de le pousser à faire progresser la recherche lexicale. En effet, même si la définition d'un mot semble être connue, il n'est pas dit qu'elle sera la même pour tous les participants, car sa compréhension varie d'un individu à l'autre. Le critère de comparaison sert également à faire ressortir des synonymes et des mots de même famille en explicitant le rapprochement de sens entre deux mots. En effet, l'analogie et la comparaison sont des moyens efficaces de rendre accessibles des concepts complexes afin de les comprendre plus facilement en utilisant le mot en question et en le comparant avec un autre mot pour en faire ressortir un lien qui les unit. De plus, la présentation d'exemples et de contre-exemples permet de mettre les mots en contexte, aidant ainsi les autres participants à mieux comprendre les différents points de vue, à nuancer leur perception et à générer rapidement des mots en lien avec une thématique. Il est possible d'être en accord ou en désaccord avec l'idée que deux mots expriment des idées contraires (p. ex. : Est-ce que les mots rechercher et éviter sont des antonymes ?). Certains peuvent trouver que des aspects du mot expriment une même idée alors que d'autres seraient opposées (p. ex. : les mots rechercher et chercher). Le fait de tenir compte du contexte et de varier les angles d'approche permet aux élèves d'approfondir leur pensée tout en générant des mots en liens avec une thématique et des antonymes. Les enfants relèvent parfois le caractère polysémique de certains mots lors des échanges. La mise en contexte par des exemples de phrases d'élèves permet de clarifier les différents sens d'un même mot. Pour éviter les malentendus, l'enseignant souligne le caractère polysémique du mot et encourage les élèves à comparer les différents usages qu'ils en font durant la discussion. Lorsque l'occasion se présente, ces 
échanges suscitent des situations d'apprentissage authentiques sur la polysémie. L'étape 2 de la démarche de la CRL, lors de la recherche du mot dans le dictionnaire, sert également à clarifier les différents sens d'un même mot et à exemplifier, au besoin à l'aide d'extraits de la discussion, les différents usages.

\section{Méthodologie}

16 L'approche méthodologique privilégiée pour ce projet de recherche relève d'un paradigme qualitatif de type descriptif-interprétatif (Paillé \& Mucchielli, 2012). Dans les faits, le cycle de recherche, réflexion et action a consisté à permettre aux enseignantes et aux élèves de s'engager à titre d'acteurs actifs tout au long du processus de recherche et de participer aux échanges en salle de classe avec les assistants et assistantes de recherche et les deux chercheuses ainsi qu'à l'extérieur de celle-ci pendant les périodes d'élaboration des différentes étapes et de développement des outils pédagogiques.

\subsection{Contexte de la recherche}

17 Le projet de recherche s'est déroulé dans une école primaire de la région métropolitaine de Montréal, dans la province de Québec. Bien que l'école se situe dans un contexte majoritairement francophone, une forte concentration pluriethnique et plurilingue caractérise la population des élèves participants. L'indice de défavorisation (indice de milieu socioéconomique et indice de revenu familial) de l'école sélectionnée se situait au seuil le plus défavorisé, soit 10 sur une échelle de 10. La défavorisation et le plurilinguisme du milieu scolaire constituaient des critères de sélection pour l'étude afin de vérifier si la démarche didactique est applicable dans un milieu où les enfants ont un moins grand bagage lexical dans la langue de scolarisation.

\subsection{Collecte des données}

Une des méthodes de collecte de données privilégiées a été l'observation ethnographique numérique (Pellerin, 2017) à l'aide d'outils technologiques comme la tablette numérique. Lors des six discussions lexicales menées, les enseignantes, une ou deux des chercheuses et une assistante à la recherche étaient présentes en classe. Elles utilisaient les tablettes numériques mises à leur disposition pour documenter le déroulement des discussions lexicales. Chaque discussion lexicale comporte plusieurs vidéos du déroulement permettant de saisir les propos de la discussion, les actions des élèves observateurs ainsi que les notes audios de l'équipe de recherche. L'observation ethnographique numérique permet de contextualiser et de nuancer certains résultats en plus de fournir des traces tangibles des discussions lexicales.

Après chacune des discussions lexicales, les cartes de mots des observateurs ont été recueillies à titre d'artéfacts produits par les élèves comme sources de données. Ces cartes témoignent des mots notés par les élèves lors de l'écoute active de la discussion lexicale. Aux fins de cet article, l'analyse du corpus de mots de la discussion lexicale à partir du mot RECHERCHER a été réalisée sur un groupe classe de $3^{e}$ année du primaire, soit 19 participants âgés entre 8 et 9 ans, divisés en deux sous-groupes (élèves interlocuteurs et élèves observateurs) qui ont alterné leurs rôles après quinze minutes 
(consigne donnée aux élèves observateurs de la discussion lexicale : Notez tous les mots ayant selon vous un lien de sens avec le mot RECHERCHER). Les 19 cartes d'observateurs produites par les participants durant cette discussion lexicale ont été analysées.

\subsection{Traitement et analyse des données}

Afin d'analyser les données issues de l'observation ethnographique numérique, nous avons procédé à la transcription intégrale de tous les mots énoncés par les 19 élèves, à partir de l'enregistrement vidéo des deux sous-groupes de discussions lexicales (sousgroupe A, 9 élèves, et sous-groupe $B, 10$ élèves). Par la suite, tous les mots notés sur chacune des cartes d'observateur ont été inventoriés. L'ensemble des données a été soumis à une analyse de type catégorielle réalisée à l'aide du logiciel NVivo, ce qui a permis le codage et le contrecodage selon les quatre catégories de relations sémantiques (mot de même famille morphologique, mot en lien avec une thématique, synonyme, antonyme). Afin de vérifier si les mots énoncés et notés par les élèves avaient ou non un lien de sens avec le mot à l'étude et d'assurer la validité des classifications établies, trois outils de référence ont été utilisés: le dictionnaire Antidote 10, le dictionnaire électronique des synonymes $C R I S C O^{10}$ et le Grand Robert des synonymes, nuances et contraires (2019). Le recours à ces trois outils a permis la mise à l'épreuve et la contrevérification des catégorisations. La quantité de mots inventoriés par catégorie de relation sémantique a permis de répondre à la question, déjà mentionnée, que nous reproduisons ici : en quoi une discussion lexicale réalisée par des élèves de 8-9 ans en contexte scolaire plurilingue au Québec dans le cadre de la CRL estelle suffisamment riche lexicalement pour amorcer un travail d'analyse des relations sémantiques?

\section{Présentation des résultats}

La figure 3 présente une carte d'observateur type pour le mot RECHERCHER. 
Figure 3. - Carte d'observateur remplie par une élève.

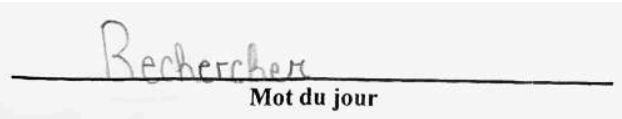

Écris des mots ayant un lien de sens avec le mot du jour

- perdue<smiles>[CH]1[CH]C=C1</smiles>

- enquête

- rechercher

- quête

- scentifique

- criminalisasion

- détective

Chercher

- inspeclever

- GPJ

- on re

- détecté

- arguélogiste

- espions

Pour le sous-groupe A $(n=9)$, les élèves ont écrit en moyenne 34 mots par carte d'observateur. Les élèves du sous-groupe $B(n=10)$ en ont pour leur part noté en moyenne 25. L'élève du groupe A qui a noté le moins de mots en a noté 20 et celui qui en a le plus noté en a noté 50 . Pour le groupe B, le plus petit nombre de mots notés par un élèves était de 14 et le plus grand nombre de 36 . Le tableau 2 présente une synthèse des résultats de l'analyse relative aux mots énoncés et notés pour les quatre catégories de relations sémantiques (mot de même famille morphologique, mot en lien avec une thématique, synonyme, antonyme) dans les deux sous-groupes (A et B). Les mots notés correspondent à la somme des mots notés à partir de ceux qui ont été entendus lors de la discussion lexicale et ceux inférés par l'élève observateur sans que ceux-ci aient été énoncés par les élèves interlocuteurs. 
Tableau 2. - Mots énoncés et notés par catégories de relations sémantiques lors de la discussion lexicale sur le mot RECHERCHER.

\begin{tabular}{lllllll} 
Sous-groupes & \multicolumn{1}{c}{ Type } & Famille & Thématique & Synonyme & Antonyme & Total \\
\hline A & énoncés & 3 & 53 & 2 & 3 & 61 \\
& énoncés et notés & $2 / 3$ & $28 / 53$ & $1 / 2$ & $1 / 3$ & $32 / 61$ \\
& notés & 2 & 53 & 5 & 3 & 63 \\
notés pas énoncés & $0 / 2$ & $25 / 52$ & $4 / 6$ & $2 / 3$ & $31 / 63$ \\
\hline B & 1 & 34 & 1 & 0 & 36 \\
& énoncés & $1 / 1$ & $25 / 34$ & $1 / 1$ & 0 & $27 / 36$ \\
& énoncés et notés & 1 & 42 & 1 & 0 & 44 \\
& notés & $0 / 1$ & $17 / 42$ & $0 / 1$ & 0 & $17 / 44$ \\
\hline A et B & notés pas énoncés & 4 & 87 & 3 & 3 & 97 \\
& énoncés et notés & $3 / 4$ & $53 / 87$ & $2 / 3$ & $1 / 3$ & $59 / 97$ \\
& notés & 3 & 94 & 7 & 3 & 107 \\
& notés pas énoncés & $0 / 3$ & $41 / 94$ & $5 / 7$ & $2 / 3$ & $48 / 107$
\end{tabular}

Les résultats présentés dans le tableau 2 montrent qu'au total, 97 mots différents comportant des liens de sens ont été énoncés par les élèves des deux sous-groupes réunis (A et B). Sur les 97 mots énoncés, 59 mots ont été à la fois énoncés et notés, ce qui correspond à $60 \%$ du corpus total ; $45 \%$ des mots ont pour leur part été notés sans avoir été énoncés par les élèves interlocuteurs, c'est-à-dire qu'ils ont été inférés par les élèves observateurs (48/107). Ainsi, un total de 107 mots notés a été à la fois relevé de la discussion et inféré par les élèves observateurs, ce qui révèle que davantage de mots ont été relevés et inférés que réellement énoncés lors de la discussion lexicale.

Bien que moins élevés, les mots énoncés par le sous-groupe B (36) témoignent que la discussion lexicale ne parvient pas à saturation après les 15 premières minutes de discussion allouées au sous-groupe A, qui en a énoncé 61 différents préalablement. En effet, rappelons que seulement les mots nouveaux énoncés et notés par le sousgroupe B ont été catégorisés, ce qui justifie le plus faible score enregistré alors que dans les faits, certains mots se répétaient entre le sous-groupe $\mathrm{A}$ et le sous-groupe $\mathrm{B}$. Néanmoins, de nombreux mots, surtout ceux appartenant à la catégorie de mots liés à une thématique, ont été générés lors de la deuxième étape de la discussion lexicale $(25 / 34)$.

À cet égard, le nombre de mots liés à une thématique énoncés et notés dépasse largement celui des trois autres catégories de relations sémantiques. En effet, un total de 87 mots liés à une thématique ont été énoncés sur un total de 97 mots, ce qui correspond à $90 \%$ du corpus de mots énoncés. Pour les mots notés, la proportion est similaire avec 94 mots sur un total de 107 mots notés, soit $88 \%$. Cette dominance des mots liés à une thématique de ce corpus se justifie par un plus large éventail de possibilités lexicales liées à cette catégorie. $61 \%$ des mots liés à une thématique énoncés ont été notés $(53 / 87)$ alors que $44 \%$ ont été pris en note sans avoir été énoncés (41/94), c'est-à-dire inférés par les élèves observateurs.

Beaucoup plus nombreux que les autres catégories, les mots liés à une thématique énoncés et notés par les deux sous-groupes ont permis une classification selon différentes sous-catégories. Ainsi, cinq sous-catégories liées à la nature thématique des mots ont été générées (domaine de recherche, outil pour faire de la recherche, 
personne qui fait la recherche, chose recherchée, verbe d'action et autres mots associés à une recherche). Le tableau 3 présente ces résultats.

Tableau 3. - Total de mots énoncés et notés par sous-catégorie de mots liés à une thématique.

\begin{tabular}{ccccccc}
\hline domaine & outil & personne & chose & verbe & autre & Total \\
\hline 10 & 28 & 9 & 55 & 23 & 3 & 128 \\
\hline
\end{tabular}

La première sous-catégorie regroupe dix mots associés à des domaines de recherche (enquête, archéologie, philosophie, science, etc.). Parmi la catégorie «outil» pour rechercher, nous retrouvons des mots comme encyclopédie, internet, GPI, carte, boussole ainsi qu'une série de logiciels, de sites web et d'applications (Siri, Google, Firefox, AllôProf, Skype, etc.). Notons que nous avons retenu ces derniers mots pour la pertinence de leur relation sémantique avec le mot RECHERCHER, bien qu'il s'agisse de noms propres. Pour la troisième catégorie, "personne ", neuf mots liés à des corps de métiers ont été identifiés (archéologue, chasseur, détective, enquêteur, inspecteur, police, policier, scientifique et espion). La catégorie "chose recherchée » est la plus importante avec un total de 55 mots couvrant un large spectre thématique (trésor, maladie, résultat, définition, fossile, chemin, OVNI, etc.). Vingt-trois verbes d'action liés au mot RECHERCHER se retrouvent également dans le corpus total de mots liés à une même thématique (calculer, discuter, refaire, questionner, remarquer, observer, etc.).

Pour ce qui est des trois autres catégories, trois des quatre mots de même famille énoncés ont également été notés (recherche, cherche, chercher). Un seul mot de même famille morphologique énoncé n'a pas été noté (recherché). Quant aux trois synonymes énoncés (enquêter, chercher, explorer), deux d'entre eux ont été notés (chercher, explorer). Notons que sept synonymes ont été pris en note sur les cartes d'observateurs, alors que cinq d'entre eux n'ont pas été énoncés par les élèves interlocuteurs (fouiller, regarder, explorer, examiner, investiguer). Seulement les synonymes chercher et enquêter ont réellement été énoncés. Finalement, sur les trois antonymes énoncés (trouver, détecter, retrouver), un seul a pu être noté (trouver) et deux ont été notés sans être énoncés (découvrir, apercevoir).

\section{Discussion des résultats}

La mise en comparaison des mots énoncés par les élèves interlocuteurs avec les mots notés par les élèves observateurs permet de révéler l'étendue lexicale suscitée par l'échange entre pairs et par l'écoute active lors d'une discussion lexicale selon l'approche dialogique proposée pour l'enseignement et l'apprentissage des relations sémantiques. Les résultats présentés sont discutés en matière de capacités de production, de saisie et d'inférence d'élèves en situation de discussion lexicale. La description des mots énoncés, notés et inférés par les élèves permet de décrire la richesse et les limites de la discussion lexicale dans le cadre de la CRL. L'analyse du corpus de mots permet de réfléchir au potentiel didactique de cette étape comme prémisse à l'enseignement des relations sémantiques. La dernière partie de la discussion analyse le potentiel didactique de la discussion lexicale. 


\subsection{Capacité à énoncer des mots par les élèves interlocuteurs}

30 L'analyse des enregistrements vidéos permet de constater que la discussion lexicale, telle qu'elle est proposée et structurée lors de la démarche didactique de la CRL, permet aux élèves de générer un grand nombre de mots ayant un lien de sens avec le mot du jour. La question du choix du mot se pose. Il est important de choisir un mot ayant une portée conceptuelle riche (cadeau, aimer, beauté, toujours, etc.) ou des mots polysémiques favorisant la discussion. Les résultats montrent que, durant les deux périodes de 15 minutes de discussion lexicale, les élèves ont énoncé un total de 97 mots différents ayant un lien de sens avec le mot RECHERCHER. Sur ces 97 mots énoncés par les deux sous-groupes, 59 mots ont été notés, ce qui signifie que les élèves observateurs parviennent à repérer et à noter de nombreux mots ayant un lien de sens en écoutant leurs pairs discuter. Rappelons que l'objectif de la discussion lexicale n'est pas de noter le plus grand nombre possible de mots, mais bien de détecter des mots ayant un lien de sens avec le mot RECHERCHER dans ce cas-ci. Ce résultat confirme que la discussion lexicale constitue l'assise permettant le travail d'analyse des relations sémantiques avec les élèves qui se déroule lors des étapes subséquentes de la démarche didactique de la CRL. Cela corrobore les propos des participants qui mentionnaient, lors d'entretiens qui ne font pas l'objet de cet article, que la discussion à plusieurs permettait l'accessibilité lexicale et le partage des idées et des mots (voir Lavoie, Pellerin, Brel-Cloutier \& Beauparlant, 2019).

31 La particularité de la discussion lexicale de la CRL, en plus d'être planifiée et structurée, consiste à partager des explications sur le sens d'un mot entre élèves de façon collaborative. En effet, les idées amenées par un élève semblent favoriser l'émergence de mots par d'autres élèves. La capacité de production lexicale se déploie et donne lieu à l'enchainement de plusieurs mots liés à un même thème. Il n'est pas étonnant que $90 \%$ des mots énoncés sont des mots appartenant au champ lexical lié au thème, car cette catégorie de lien sémantique est nécessairement plus riche que les synonyme/ antonyme/famille morphologique. Par exemple, un élève énonce d'abord le mot espion, ce qui donne lieu à une série de mots du même champ sémantique (détective, inspecteur, policier, criminel, criminalité, voleur, etc.). Cet exemple n'est pas unique, lorsque nous analysons le déroulement d'une discussion lexicale ${ }^{11}$, nous constatons que cette approche discursive favorise, grâce au groupe, la production de nombreux mots permettant l'approfondissement lexical. Ces résultats corroborent les affirmations de Nonnon $(1996,2008)$ et de Mazière (1993), à savoir que la pratique de discussion en groupe favorise le déploiement d'une variété de mots et le partage des expériences et des références liées au mot à l'étude. Il convient de préciser que l'objectif premier de l'approche dialogique de la CRL préconisée dans cette étude est d'approfondir ensemble le sens d'un mot et les liens lexicaux qui unissent le mot aux autres (Lavoie, à paraitre).

Les résultats de l'étude indiquent que la discussion lexicale favorise une production suffisante de mots pour permettre un travail subséquent sur l'acquisition des notions par les élèves. De plus, nous remarquons que les classes des mots produits sont variées. Les noms communs demeurent néanmoins dominants, surtout en ce qui a trait aux mots liés à une thématique. La quasi-totalité des mots produits par les participants est constituée des mots fréquents du registre de langue courant de la langue française. Certains renvoient toutefois à un registre plus soutenu (p.ex.: archéologique, philosophique, criminalité, etc.). Il est important de rappeler que le contexte 
socioéconomique des élèves influe sur leur bagage lexical (Hart \& Risley, 1995), mais qu'à plusieurs, ils réussissent à produire et à noter suffisamment de mots pour amorcer un travail lexical sur les relations sémantiques. Lors des étapes subséquentes, ils utiliseront des outils lexicaux (dictionnaire, arbre de décisions lexicales) leur permettant de poursuivre seul, avec leurs pairs et avec l'enseignante, leur travail d'analyse et de recherche de mots ayant des liens de sens. Notre étude indique que la discussion lexicale permet de déployer suffisamment de mots de chaque catégorie de relations sémantiques, et ce, même dans un contexte plurilingue où les apprenants n'ont pas la langue de scolarisation comme langue première.

La discussion lexicale correspond à une " tâche discursive ", telle qu'elle est décrite par Grandaty (2006), car l'enseignante oriente la discussion par des questions et des relances, favorise les échanges entre pairs, implique des négociations et des prises de conscience sur l'activité disciplinaire et transforme sur la durée la posture des apprenants face à l'apprentissage lexical. Par exemple, elle peut faire explorer la notion de polysémie en faisant comparer différents sens d'un mot utilisé par différents enfants. L'enseignante guide les échanges, tout en acceptant que les apprenants partent de leurs représentations du mot pour coconstruire le sens. Nos résultats rappellent que le travail de synthèse, de relance et de questionnement ${ }^{12}$ de l'enseignante suscite la production de mots des élèves. Voici un exemple tiré de la transcription verbatim du sous-groupe A :

E1 : Tu es en train de dire qu'il y a des personnes qui sont spécialisées pour rechercher des choses en particulier?

é6 : oui, comme le détective ou l'inspecteur.

\subsection{Capacité à noter les mots ayant un lien de sens par les élèves observateurs}

L'analyse des mots notés permet de constater les capacités d'écoute active, de saisie et de discrimination des élèves observateurs. Le fait de compléter une carte de mots comme tâche d'écoute rend actifs les observateurs de la discussion (Lavoie, Pellerin, Brel-Cloutier \& Beauparlant, 2019). Plusieurs raisons expliquent le fait que tous les mots ayant un lien de sens n'aient pas été notés, car le nombre de mots liés à une thématique énoncés dépassait largement ce dont les apprenants avaient besoin pour remplir leur carte lexicale heuristique lors de l'étape suivante de la démarche didactique. De plus, la vitesse des échanges contribue au fait que les apprenants ne pouvaient pas noter tous les mots entendus. Les résultats du tableau 2 révèlent une bonne capacité à discriminer les mots ayant des liens de sens.

\subsection{Capacité d'inférence des élèves observateurs lors de l'écoute active des mots}

La capacité d'inférence des élèves observateurs les mène à noter des mots sur leur carte lexicale sans les avoir nécessairement entendus. Les liens de sens se font sur le plan cognitif, dans leurs représentations internes, et leur saisie sur la carte lexicale rend explicite ce chemin inférentiel (Aitchison, 2012). L'écoute active des élèves observateurs favorise le déploiement de leur bagage lexical. En effet, à l'instar de la théorie socioculturelle, la discussion lexicale constitue une forme de participation active qui engage tant les interlocuteurs que les observateurs dans une posture 
d'écoute active qui les mène à faire des liens référentiels avec le mot entendu. À notre avis, ces résultats peuvent varier d'une CRL à une autre, selon le profil des apprenants qui composent le groupe, les conditions dans lesquelles s'exerce la discussion lexicale, mais surtout selon l'étendue du champ lexical du mot déterminé pour mener une CRL. À cet égard, Mondada et Pekarek Doehler (2004) avancent que le sens des mots se construit en interaction avec les autres. Cette affirmation fait écho aux résultats obtenus dans la présente étude qui mentionnent que la discussion lexicale en groupe permet d'avoir plus d'idées (Lavoie, Pellerin, Brel-Cloutier \& Beauparlant, 2019). À travers l'interaction émerge une curiosité pour les mots ainsi qu'un esprit collaboratif pour chercher, pour négocier, pour coconstruire, pour trouver et pour approfondir le sens des mots.

\subsection{Potentiel de la discussion lexicale pour l'enseignement et l'apprentissage des relations sémantiques}

36 À la lumière des résultats dégagés, la discussion lexicale se présente comme une tâche discursive collaborative qui installe un climat d'entraide, d'écoute et de partage au sein du groupe classe, créant une communauté d'apprentissage pour l'apprentissage du lexique. Ce climat favorise le travail d'enseignement explicite de stratégies lexicales qui s'opérationnalise lors de la troisième étape de la démarche didactique (voir fig. 1). C'est ce corpus de mots produits par les élèves qui, dans un second temps, guide l'étape de la modélisation des stratégies lexicales. En ce sens, la démarche de la CRL répond à trois composantes d'un enseignement lexical efficace selon Graves (2006), car elle offre une expérience de langage riche et variée, elle soutient l'enseignement de mots précis et elle propose des stratégies lexicales aux élèves. Tel qu'il est préconisé dans la discussion philosophique (Sasseville \& Gagnon, 2012), la discussion lexicale permet également de développer leur autonomie et leur discernement.

Dans le cadre de cette étude menée en contexte plurilingue, nous avons pu observer la nécessité d'entamer le travail lexical avec le dialogue afin de créer un contexte d'apprentissage inclusif. À cet égard, les écrits plus récents de Van Compernolle (2015) réitèrent l'importance d'amorcer le travail sémantique à partir des représentations et du vécu des élèves au moyen du dialogue. Dans la démarche de la CRL, nous constatons que le discours dialogique lors de l'étape de la discussion lexicale permet de faire émerger les représentations des élèves sur le mot cible (Mondada \& Pekarek Doehler, 2004 ; Van Compernolle, 2015) tout en reflétant la pluralité de leur bagage lexical et expérientiel. Dans le cadre d'une étude ultérieure, il serait intéressant de nous attarder plus spécifiquement au rôle que joue l'enseignante lors d'une discussion lexicale et aux effets de ses questionnements et de ses relances sur le travail des apprenants.

\section{Conclusion}

La communauté de recherche lexicale (CRL) est une démarche didactique dialogique au service de l'enseignement et de l'apprentissage des relations sémantiques. Conçue pour les élèves de l'école primaire âgés de 8 à 12 ans, elle a été développée dans le cadre d'un projet de recherche-action mené en contexte scolaire plurilingue au Québec. La CRL comporte cinq étapes en étroite interdépendance les unes des autres. Cet article a traité exclusivement de la première étape de la discussion lexicale, laquelle s'inspire 
d'avancées réalisées dans le champ de la philosophie pour enfants et prend appui sur la théorie socioculturelle des interactions ainsi que sur les approches dialogiques. L'apprentissage s'y réalise dans l'action, à travers la prise de parole, l'écoute active et la prise de notes. Les résultats révèlent le potentiel de la discussion lexicale pour la production d'un corpus de mots authentiques selon quatre catégories de relations sémantiques (mot de même famille morphologique, mot en lien avec une thématique, synonyme, antonyme). Cette étape constitue une situation d'apprentissage authentique permettant d'énoncer, de saisir et d'inférer des mots ayant des liens de sens entre eux. Faisant écho aux recherches dans le domaine, les résultats démontrent que l'interaction suscitée par la discussion lexicale, guidée par les questions de l'enseignante, favorise la verbalisation de la pensée, l'expression des représentations internes des apprenants ainsi que le déploiement d'une étendue lexicale suffisamment riche pour amorcer un travail d'analyse, ce qui confirme l'importance des approches dialogiques pour soutenir l'enseignement et l'apprentissage du lexique à l'école primaire.

\section{BIBLIOGRAPHIE}

Aitchison, Jean. (2012). Words in the Mind: An Introduction to the Mental Lexicon (4 ${ }^{\mathrm{e}}$ éd.). Chichester, Royaume-Uni : Wiley-Blackwell.

AMER, Aly Anwar. (2002). Advanced Vocabulary Instruction in EFL. The Internet TESL Journal, 8(11). Disponible en ligne sur <http://iteslj.org/Articles/Amer-Vocabulary/>.

ANCTIL, Dominic. (2019). Comment enseigne-t-on le lexique dans les classes au primaire ? Vivre le primaire, 32(1), 14-17.

AnCTIL, Dominic, SingCASTER, Mélissa \& TARDIF, Mylène. (2018). Pratiques d'enseignement du lexique en classe de primaire au Québec. La lettre de l'AIRDF, 64, 19-25.

BENGOCHEA, Alain (2014). The Effects of Vocabulary Instruction on Bilingual Students' Lexico-Semantic Acquisition in English-MediumContexts: A Systematic Analysis. Open Access Dissertations. Paper 1322. Disponible en ligne sur <https://scholarship.miami.edu/discovery/fulldisplay/ alma991031447776802976/01UOML_INST:ResearchRepository>.

CALAQUE, Elizabeth. (2000). Enseignement et apprentissage du vocabulaire, hypothèse de travail et propositions didactiques. Lidil, 21, 17-35.

COMMUNAUTÉ DE RECHERCHE LEXICALE. (2020). Communauté de recherche lexicale. Disponible en ligne sur <http://crl.recherche.usherbrooke.ca> (consulté le 9 août 2020).

DANIEL, Marie-France, LAFORTUNE, Louise, PALLASCIO, Richard \& SYKES, Pierre. (2004). Philosopher sur les mathématiques et les sciences. Québec, Canada : Le Loup de Gouttière.

DREYFUS, Martine. (2004, août). Pratiques et représentations de l'enseignement du lexique à l'école primaire. Communication présentée au $9^{e}$ colloque de l'Association internationale pour la recherche en didactique du français (AIRDF), Québec, Canada. Disponible en ligne sur <http:// www.colloqueairdf.fse.ulaval.ca/fichier/Communications/martine-dreyfus.pdf>. 
DuvignaU, Karine \& GARCIA-DEBANC, Claudine. (2008). Un apprentissage du lexique verbal par proximité sémantique : quand la représentation lexicale facilite la tâche d'écriture. Dans F. Grossmann \& S. Plane (dir.), Les apprentissages lexicaux : lexique et production verbale (p. 17-41). Villeneuve : Presses universitaires du Septentrion. <https://doi.org/10.4000/books.septentrion. 14522>.

EDMONDS, Philip \& HIRST, Graeme. (2002). Near-Synonymy and Lexical Choice. Computational Linguistics, 28(2), 105-144. <https://doi.org/10.1162/089120102760173625>.

FIRTH, Alan \& WAGNER, Johannes. (1997). On Discourse, Communication, and (Some) Fundamental Concepts in SLA Research. The Modern Language Journal, 81(3), 285-300.

GAGNON, Mathieu. (2012). La pratique enseignante du dialogue philosophique en communauté de recherche. Dans N. Bouchard \& M. Gagnon (dir.), Éthique et culture religieuse en question : réflexions critiques et prospectives (p. 65-84). Québec, Canada : Presses de l'Université du Québec.

Goodwin, Marjorie Harness \& KYratzIs, Amy. (2007). Children Socializing Children: Practices for Negotiating the Social Order Among Peers. Research on Language \& Social Interaction, 40(4), 279-289. <https://doi.org/10.1080/08351810701471260>.

MINISTÈRE DE L'ÉDUCATION, DU LOISIR ET DU SPORT (MELS). (2011). Progression des apprentissages. Québec, Canada : Gouvernement du Québec.

GRANDATY, Michel. (2006). Place et rôle des conduites discursives orales dans le système des médiations en classe : des objets travaillés aux objets enseignés ? Dans B. Schneuwly (dir.), Analyses des objets enseignés : le cas du français (p. 93-110). Louvain-la-Neuve, Belgique : De Boeck Supérieur. <https://doi.org/10.3917/dbu.schne.2006.01.0093>.

Grand Robert des synonymes, nuances et contraires. (2019). Paris, France : Éditions Le Robert. GRAVES, Michael F. (2006). The Vocabulary Book. Learning \& Instruction. New York, NY : Teachers College, Columbia University.

HART, Betty \& RISLEY, Todd R. (1995). Meaningful Differences in the Everyday Experience of Young American Children. Baltimore, MD : Paul H. Brookes Publishing Company.

LAVoIE, Constance. (À paraitre). L'effet de la tablette tactile sur l'acquisition des relations sémantiques. Revue canadienne de linguistique appliquée, 23(1).

Lavoie, Constance, Pellerin, Martine, Brel-Cloutier, Anne \& Beauparlant, René. (2019). La communauté de recherche lexicale : perceptions des élèves et des enseignants sur une démarche dialogique et multimodale d'enseignement-apprentissage des relations sémantiques. Dans G. Messier \& L. Lafontaine (dir.), Littératie : entre pratiques scolaires et extrascolaires (p. 57-85). CôteSaint-Luc, Canada : Peisaj.

LAVoie, Constance, Pellerin, Martine \& GiRARD, Kevin. (2017). La communauté de recherche lexicale : une démarche dialogique et multimodale au service de l'enseignement du lexique. Vivre le primaire, 30(3), 15-22. Disponible en ligne sur <http://crl.recherche.usherbrooke.ca/wpcontent/uploads/2018/07/revue-VLP-AQEP-303-VF_15-19_2.pdf>.

LIPMAN, Matthew, SHARP, Ann Margaret \& OsCANYAN, Frederick. S. (1980). Philosophy in the Classroom. Philadelphie, PA : Temple University Press.

MAZIÈRE, Francine. (1993). Le mot, unité didactique : une entrée dans la langue par le mot. Repères, 8, 29-39. 
MondADA, Lorenza \& PeKAREK Doehler, Simona. (2004). Second Language Acquisition as Situated Practice: Task Accomplishment in the French Second Language Classroom. The Modern Language Journal, 88, 501-518.

NONNON, Elisabeth. (2008). Apprendre des mots, construire des significations : la notion de polysémie à l'épreuve de la transdisciplinarité. Dans F. Grosmann \& S. Plane (dir.), Les apprentissages lexicaux (p. 43-69). Paris, France : Presses universitaires du Septentrion.

NonNON, Elisabeth. (1996). Activités argumentatives et élaboration de connaissances nouvelles : le dialogue comme espace d'exploration, Langue française, 1, 67-87. <https://doi.org/10.3406/lfr. 1996.5361>.

PAILlÉ, Pierre \& Mucchielli, Alex. (2012). L'analyse qualitative en sciences humaines et sociales. Paris, France : Armand Colin. <https://doi.org/10.3917/arco.paill.2012.01>.

PelLeRIN, Martine. (2017). La pensée médiatisée au service du développement des habiletés de régulation métacognitive chez les élèves. Dans C. Dumais, R. Bergeron, M. Pellerin \& C. Lavoie (dir.), L'oral et son enseignement : pluralité des contextes linguistiques (p. 221-242). Montréal, Canada : Peisaj.

Plane, Sylvie \& LAfourCADE, Bernadette. (2004). Pour une approche discursive de l'apprentissage du lexique : les activités définitionnelles. Dans E. Calaque (dir.), Didactique du lexique : contextes, démarches, supports (p. 47-59). Louvain-la-Neuve, Belgique : De Boeck Supérieur. <https://doi.org/ 10.3917/dbu.didle.2004.01.0047>.

POLGUÈRE, Alain. (2016). Lexicologie et sémantique lexicale. Notions fondamentales ( $3^{\mathrm{e}}$ éd.). Montréal : Les Presses de l'Université de Montréal. Disponible en ligne sur <http://books.openedition.org/ pum/23829>.

SASSEVILLE, Michel \& GAGNON, Mathieu. (2012). Penser ensemble à l'école, des outils pour l'observation d'une communauté de recherche philosophique en action. Québec, Canada : PUL.

VAN COMPERNOLLE, Rémi. (2015). Interaction and Second Language Development: A Vygotskian Perspective. Amsterdam, Pays-Bas : John Benjamins.

Vygotsky, Lev. (1987). Pensée et langage. Paris, France : Les Éditions sociales.

\section{NOTES}

1. Voir <www.uqac.ca/crl/index.php/etape-1/>.

2. Voir <www.uqac.ca/crl/index.php/etape-1/>.

3. Voir <www.uqac.ca/crl/index.php/etape-2/>.

4. Voir <www.uqac.ca/crl/index.php/etape-3/>.

5. Voir <http://crl.recherche.usherbrooke.ca/index.php/etape-3/>.

6. Voir <www.uqac.ca/crl/index.php/etape-4/>.

7. Voir <www.uqac.ca/crl/wp-content/uploads/2018/07/Carte-lexicale-heuristique.jpg>.

8. Voir <www.uqac.ca/crl/index.php/etape-5/>.

9. Voir < www.uqac.ca/crl/wp-content/uploads/2018/08/Guide-de-discussion-structurée-pourle-mot-rechercher.pdf>. Ce guide de discussion est celui pour le mot RECHERCHER. L'équipe de la CRL a élaboré un guide de discussion pour chaque mot cible, dans lequel sont incluses des questions pour guider l'animation de la discussion lexicale et des suggestions de romans philosophiques ou d'albums jeunesse. En complément du guide d'animation vient une grille d'observation pour évaluer les apprenants lors de la discussion lexicale. 
10. Voir <https://crisco2.unicaen.fr/des/>.

11. Voir <www.uqac.ca/crl/index.php/etape-1/>.

12. Voir <http://crl.recherche.usherbrooke.ca/index.php/etape-1/>.

\section{RÉSUMÉS}

L'apprentissage des relations sémantiques est une tâche complexe qui nécessite de tisser des liens de sens en partant de mots connus et des représentations initiales des apprenants. Or, peu d'enseignement systématique du lexique et des relations sémantiques serait réalisé en classe et le potentiel des approches discursives pour cet apprentissage serait peu exploité. Devant le manque de ressources didactiques et en nous inspirant d'avancées réalisées dans le champ de la philosophie pour enfants, nous avons développé la démarche de la communauté de recherche lexicale (CRL). Prenant appui sur les approches dialogiques et la théorie socioculturelle des interactions, cette démarche didactique a émergé d'un projet de recherche-action mené par deux chercheuses canadiennes auprès d'élèves et d'enseignantes d'une école primaire située en contexte plurilingue au Québec. Le présent article explore de façon plus approfondie la première étape $^{1}$ de la démarche, laquelle s'opérationnalise par une discussion lexicale sur le sens d'un mot et l'écoute active des mots. L'apprentissage s'y réalise dans l'action, à travers la prise de parole, l'écoute active et la prise de notes. Faisant écho aux recherches dans le domaine, l'interaction suscitée par la discussion lexicale, guidée par les questions de l'enseignante, favorise la verbalisation de la pensée et l'expression des représentations internes des apprenants ainsi que le déploiement d'une étendue lexicale propice à l'amorce d'un travail d'analyse des relations sémantiques. La discussion lexicale constitue donc une situation d'apprentissage authentique permettant d'énoncer, de saisir et de trouver des mots connus qui entretiennent des liens de sens avec un mot cible. Ces résultats confirment l'importance des approches dialogiques pour soutenir l'enseignement du lexique à l'école primaire.

Learning semantic relationships is a complex task that requires making connections of meaning from known words and learners' initial representations. However, little systematic teaching of the lexicon and semantic relations would be carried out in the classroom and the potential of discursive approaches for this learning would be little exploited. Faced with the lack of teaching resources and drawing inspiration from the advances made in the field of philosophy for children, we have developed the lexical research community (CRL) approach. Based on dialogical approaches and the sociocultural theory of interactions, this didactic approach emerged from an action-research project led by two Canadian researchers with students and teachers from an elementary school located in a plurilingual context in Quebec. This article explores in more depth the first step of the process, which is operationalized by a lexical discussion of the meaning of a target word. Learning takes place in action, through speaking, active listening and note taking. Echoing research in the field, the interaction generated by lexical discussion, guided by the teacher's questions, promotes the verbalization of thought and the expression of internal representations of learners as well as the deployment of lexical scope conducive to initiating analytical work. Lexical discussion therefore constitutes an authentic learning situation allowing to state, grasp and find known words which have meaningful links with a target word. These results confirm the importance of dialogical approaches to support lexicon teaching at primary school. 
INDEX

Mots-clés : didactique du français, enseignement du lexique, relations sémantiques, approches dialogiques

Keywords : didactics of French, lexicon teaching, semantic relationships, dialogical approaches

\section{AUTEURS}

\section{CONSTANCE LAVOIE}

Professeure, Université de Sherbrooke

constance.lavoie2@USherbrooke.ca

\section{PATRICIA-ANNE BLANCHET}

Doctorante, Université de Sherbrooke

patricia-anne.blanchet@usherbrooke.ca

\section{MARTINE PELLERIN}

Professeure, Université de l'Alberta (Faculté Saint-Jean)

pellerin@ualberta.ca 